\title{
Efectos de la utilización de la Inteligencia Competitiva en Pymes industriales
}

\author{
Emilio Placer-Maruri(iD) ${ }^{1}$, Daniel Pérez-González (DiD ${ }^{1}$, Pedro Soto-Acosta (iD)² \\ ${ }^{1}$ Universidad de Cantabria (Spain) \\ ${ }^{2}$ Universidad de Murcia (Spain)
}

emilio.placer@unican.es,daniel.perez@unican.es,psoto@um.es

Received January, 2016

Accepted May, 2016

\section{Resumen}

Objeto: La literatura sobre Inteligencia competitiva, como proceso avanzado de gestión de información orientado a apoyar la toma de decisiones estratégicas, se ha centrado hasta el momento en temas relacionados con la definición del concepto y en analizar mediante estudios de casos su aplicación y los resultados obtenidos en grandes empresas y gobiernos. Reconociendo la literatura que la evolución de este tema requiere avanzar en el estudio ampliándolo a otros tipos de organizaciones y profundizar en los efectos que produce la Inteligencia Competitiva. En este sentido, el presente trabajo tiene por objeto analizar en una muestra de pymes los efectos que la utilización de las IC tiene en distintas variables financieras y no financieras.

Diseño/metodología/enfoque: El trabajo sigue el esquema clásico de investigación con revisión de la literatura, proposición de hipótesis y aplicación de metodología empírica cuantitativa, recogiendo información mediante cuestionarios enviados por correo electrónico, para el posterior tratamiento y contraste estadístico mediante modelos ANOVA, que permiten obtener resultados y conclusiones. 
Aportaciones y resultados: El trabajo aporta un enfoque sobre los efectos de la IC en las empresas que van más allá de los predominantes estudios de casos y de las grandes organizaciones. Así, presenta como aportaciones analizar los efectos de la IC en una muestra de pymes y el hacerlo con una metodología empírica cuantitativa buscando relaciones causales mediante variables financieras y no financieras y contrastes de hipótesis. Los resultados obtenidos muestran que la Inteligencia Competitiva mejora la capacidad de adaptación al entorno de las pymes, su capacidad de innovar y contribuye a la generación de valor en el negocio.

Limitaciones: La falta en la literatura de trabajos previos centrados en muestras de pymes que permitan tanto servir de guía como establecer comparaciones con el trabajo aquí desarrollado. El tamaño muestral (79 empresas) dificulta la extrapolación de resultados. Además, a futuro sería adecuado identificar más variables de negocio y analizar distintos sectores de actividad.

Originalidad / Valor añadido: Este trabajo constituye un paso adelante en la medición de los efectos generados por la Inteligencia Competitiva en las pymes, que permite constatar lo que hasta el momento solo se había analizado con estudios de casos. Además incorpora un enfoque con variables financieras y no financieras que pueden orientar tanto futuras investigaciones, como a las empresas que quieran generar su propio sistema de autoevaluación.

Palabras clave: Inteligencia Competitiva, Pymes, Indicadores cuantitativos y cualitativos, Resultados empresariales

Códigos JEL: D83, O30

Title: Effects of the use of competitive intelligence in industrial SMEs

\section{Abstract}

Purpose: The literature about Competitive Intelligence (CI), as an advanced process of information management orientated towards supporting strategic decision making, has focused on topics related to the definition of the concept and on analyzing its application and the results obtained by large firms and governments through case studies. The literature recognizes that fact that the evolution of this topic creates a need to delve deeper in its study while applying it to other types of organizations and studying the effects that Competitive Intelligence produces 
in greater depth. Therefore, this study's objective is to analyze the effects that the use of CI has on different financial and not financial variables within a sample of SMEs.

Design/methodology: The work follows the classical research scheme with literature review, statement of hypotheses and application of quantitative empirical methodology, collecting information through questionnaires sent by email, for further processing and statistical testing using ANOVA models, which allow get results and conclusions.

Findings: This study's contribution comes from providing a new way to focus on the effects of de CI in companies and goes further than most of the studies cases and big companies. Therefore, its contributions are to analyze the effect of the CI within a group of SMEs and, using an empiric methodology, to look for causal relationships through financial and nonfinancial variables and tests on the hypotheses. The results show that Competitive Intelligence improves SMEs' adaptation capacity to their environment, improves their innovation capacity and supports value creation in their businesses.

Research limitations/implications: The lack in the literature from previous studies that focused on samples of SMEs makes it possible to provide guidance and to make comparisons with the work that we carried out here. The sample size (79 companies) makes it difficult to extrapolate results. In addition, in the future it would be more appropriate to identify and analyze variables of different business sectors.

Originality/value: This work provides a step forward in measuring the effects generated by Competitive Intelligence in SMEs, making it possible to demonstrate that only case studies have been analyzed. It also incorporates an approach using financial and non-financial variables that can guide future research as well as companies that want to generate their own self-assessment systems.

Keywords: Competitive Intelligence, SME's, Quantitative and qualitative result indicators

Jel Codes: D83, O30 


\section{Introducción}

Desde hace tiempo tanto en el ámbito académico como en el empresarial se viene hablando de la importancia de la adecuada gestión de la información, del conocimiento y de las tecnologías de la información (en adelante TI) como vías para mejorar la eficiencia y competitividad de las organizaciones (Powelt \& Dent-Micallef, 1997; Dedrick, Gurbaxani \& Kraemer, 2003; Kohli \& Grover, 2008; Trigueros-Preciado, Pérez-González \& Solana-González, 2013; Pérez-González \& Díaz-Díaz, 2015), sin embargo la dureza de la crisis y sus efectos especialmente en las pequeñas y medianas empresas (en adelante Pymes) del sur de Europa, donde de media en esta crisis han desaparecido el 20\% de las empresas (Eurofound, 2013; OECD, 2015) han puesto de manifiesto que todavía queda mucho por hacer en esta materia.

En estos contextos de dificultades y complejidad donde adquieren una especial importancia conceptos y herramientas del management como la Inteligencia Competitiva (en adelante IC), proceso de gestión de la información, tecnológico, estratégico y organizativo, que permite poner en tiempo y forma a disposición de quienes tienen que tomar decisiones un conjunto de informaciones que facilitan detectar con anticipación los posibles cambios en el entorno que puedan afectar a la empresa, además de favorecer la generación de conocimiento para el desarrollo de nuevos o mejorados productos y procesos (Escorsa \& Maspons, 2001; Brody, 2008; Gaidelys \& Meidute, 2012; Donohue \& Murphy, 2016). Conceptos y herramientas que no son nuevos si bien mayoritariamente su estudio en el management se ha centrado en analizar su aplicación en grandes empresas y gobiernos mediante casos de estudio y en el ámbito anglosajón, revelando el análisis de la literatura una importante carencia de trabajos que analicen los efectos que la utilización de la IC puede generar en las pymes (Attaway, 1998; Brody, 2008; Aspinall, 2011; Bulger, 2013).

Es necesario abordar este déficit por cuanto que las pymes son las organizaciones empresariales más numerosas en las economías desarrollas, donde constituyen más del $90 \%$ del total de las empresas (OECD, 2015) y especialmente en España donde representan el 99\% del tejido empresarial (Dirección General de Industria y de la PYME, 2015). De ahí que organismos como la OCDE (2009) y European Comission (2015) insistan en la necesidad de desarrollar investigaciones y políticas que estudien las cuestiones que faciliten la sostenibilidad y competitividad de las pymes.

Ante la situación descrita, el objetivo de este trabajo es analizar en pymes los resultados que se pueden obtener de la aplicación de la IC. Para ello, en primer lugar se analizarán los antecedentes y el marco 
teórico sobre el que se apoya la investigación. A continuación, se presentará la metodología aplicada en el trabajo, para finalizar con el análisis de resultados y exposición de las conclusiones.

\section{Revisión de la literatura}

El concepto de IC y su aplicación al managment como herramienta de apoyo a la toma de decisiones estratégicas tiene sus orígenes en los años 80 en Estados Unidos, aumentando su repercusión con el nacimiento en 1986 de la Society for Competitive Intelligence Professionals. Desde entonces el concepto ha ido evolucionando apareciendo en la literatura con múltiples denominaciones como "Inteligencia de Negocios" (Pearce, 1976; Gile, Kirby, Karel, Teubner, Driver \& Murphy, 2006), "Análisis de la Competencia" (Ghoshal \& Westney, 1991), "Inteligencia de Mercado", (Maltz \& Kohli, 1996), "Vigilancia Tecnológica" (Palop \& Vicente, 1999; Rovira, 2008) hasta la actualidad donde la acepción más utilizada en la literatura es el término de IC, por considerar que engloba todos los demás (Calof \& Wright, 2008; AENOR, 2011; Luu, 2013; Yin, 2015) y presentando múltiples definiciones, variantes unas de otras como se puede ver en la Tabla 1.

\begin{tabular}{|c|c|}
\hline Descripción & Autor/ año \\
\hline $\begin{array}{l}\text { La IC es el sistema de aprendizaje sobre las capacidades y comportamientos de los } \\
\text { competidores actuales y potenciales con objeto de ayudar a los responsables en la } \\
\text { toma de decisión estratégica. }\end{array}$ & Cleland \& King (1975) \\
\hline $\begin{array}{l}\text { La IC es el proceso de obtención, análisis, interpretación y difusión de } \\
\text { información de valor estratégico sobre la industria y los competidores, que se } \\
\text { transmite a los responsables de la toma de decisión en el momento oportuno. }\end{array}$ & Prescott (1989) \\
\hline $\begin{array}{l}\text { La IC es un sistema organizado de observación y análisis del entorno, tratamiento } \\
\text { y circulación interna de los hechos observados y posterior utilización en la } \\
\text { empresa. }\end{array}$ & Palop y Vicente (1999) \\
\hline $\begin{array}{l}\text { La IC es un programa sistemático y ético para recolectar, analizar y ponderar } \\
\text { información que puede afectar a una compañía. } \\
\text { La IC capacita a los gerentes de las compañías, con independencia de su tamaño, } \\
\text { para tomar decisiones sobre cualquier aspecto que afecte a la organización, desde } \\
\text { marketing e I+D, a cuestiones tácticas y estrategias de negocios a largo plazo. }\end{array}$ & Miller (2001) \\
\hline $\begin{array}{l}\text { La IC consiste en realizar de forma sistemática la captura, el análisis, difusión y la } \\
\text { explotación de las informaciones técnicas útiles para la supervivencia y el } \\
\text { crecimiento de la empresa. La vigilancia debe alertar sobre cualquier innovación } \\
\text { científica o técnica susceptible de crear oportunidades o amenazas. }\end{array}$ & $\begin{array}{l}\text { Escorsa y Maspons } \\
(2001)\end{array}$ \\
\hline $\begin{array}{l}\text { La IC es proceso de gestión de información legal y ético, que no tiene nada que } \\
\text { ver con el espionaje industrial ni vulnera la ética empresarial. La IC se puede } \\
\text { considerar como un proceso dentro de la gestión del conocimiento. }\end{array}$ & $\begin{array}{l}\text { Chen, Chan y Zeng } \\
(2002)\end{array}$ \\
\hline $\begin{array}{l}\text { La IC como una función que consiste: en analizar el comportamiento innovador } \\
\text { de los competidores directos e indirectos, en explorar todas las fuentes de } \\
\text { información (libros, literatura gris, oficinas de patentes, etc...), en examinar los } \\
\text { productos existentes en el mercado (análisis de la tecnología incorporada) y en } \\
\text { asistir a ferias, congresos para posicionarse respecto de los competidores y tomar } \\
\text { así conocimiento de las tecnologías que predominarán en el futuro. }\end{array}$ & Morcillo (2003) \\
\hline
\end{tabular}




\begin{tabular}{|l|l|}
\hline Descripción & \multicolumn{1}{|c|}{ Autor/ año } \\
\hline $\begin{array}{l}\text { La IC es un método sistemático de planificación, recuperación, análisis, archivo, } \\
\text { distribución de la información y de la mejora de la competitividad de las empresas } \\
\text { y organizaciones. }\end{array}$ & Tena y Comai (2004) \\
\hline $\begin{array}{l}\text { La IC es la recopilación legal y ética, análisis y distribución de información } \\
\text { relacionada al ambiente competitivo, capacidades, vulnerabilidades e intenciones } \\
\text { de los competidores del negocio. }\end{array}$ & Antia y Hesford (2007) \\
$\begin{array}{l}\text { Una de las preocupaciones clave de la IC es cubrir los agujeros negros que llevan a } \\
\text { malos entendidos sobre cómo funcionan los mercados, que está haciendo la } \\
\text { competencia, que es lo que desean los competidores o en que descansa el futuro. }\end{array}$ & \\
\hline $\begin{array}{l}\text { Estos autores definen IC "como el proceso por el cual las organizaciones obtienen } \\
\text { información acerca de competidores y del ambiente competitivo e idealmente, la } \\
\text { aplica a sus procesos de planificación y toma de decisiones para mejorar el } \\
\text { desempeño de las empresas. }\end{array}$ & $\begin{array}{l}\text { Blenkhorn y Fleisher } \\
\text { La IC es el proceso de monitoreo del ambiente competitivo que capacita a los } \\
\text { gerentes a tomar decisiones basadas en información. IC es un proceso continuo } \\
\text { que incluye la recopilación ética y legal de información, su análisis y su } \\
\text { diseminación controlada, enviándola solo a los responsables de tomar decisiones } \\
\text { para evitar que se tomen decisiones equivocadas. }\end{array}$ \\
\hline $\begin{array}{l}\text { La IC es un proceso organizado, selectivo y sistemático, para captar información } \\
\text { del exterior y de la propia organización sobre ciencia y tecnología, Seleccionarla, } \\
\text { analizarla, difundirla y comunicarla, para convertirla en conocimiento con el fin de } \\
\text { tomar decisiones con menor riesgo y poder anticiparse a los cambios. }\end{array}$ & Benody (2008) \\
\hline $\begin{array}{l}\text { Plantean un mapa estratégico de IC en el estado de desarrollo, profesionalización y } \\
\text { optimización considerando las siguientes categorías funcionales que conforman un } \\
\text { programa de calidad de IC: usuarios y usos, personas y su desarrollo profesional, } \\
\text { fuentes y métodos y políticas, procesos y procedimientos que unifican el programa } \\
\text { y aseguran que correrá suavemente }\end{array}$ & Herring y Leavitt (2011) \\
\hline
\end{tabular}

Tabla 1. Principales definiciones de la IC

En cuanto a la definición de IC, como se ha comentado en la Tabla 1, no hay una definición única. Si bien, como síntesis de las definiciones anteriores y destacando aquello en lo que principalmente coindicen podemos dar una definición propia que caracterice a la IC. En este sentido la IC se caracteriza por ser un proceso flexible y adaptable a las organizaciones en las que se desarrolla, que de forma sistemática y con una orientación tanto de gestión de información como de gestión de empresa, mediante distintas técnicas y tecnologías detecta, organiza y analiza información del entorno que es de interés para la estrategia de la empresa y que pone a disposición de la dirección con una orientación a la obtención de resultados (Prescott, 1989; Palop \& Vicente, 1999; Escorsa \& Maspons, 2001; Morcillo, 2003; Antia \& Hesford, 2007; Blenkhorn \& Fleisher, 2007; Brody, 2008; AENOR, 2011; Luu, 2013).

Respecto al estudio de la IC en el management, las primeras referencias que hacen mención a la IC en este ámbito se centran principalmente en describir que es la IC y que debe permitir a las organizaciones (Cleland \& King, 1975; Prescott, 1989; Attaway, 1998; Palop \& Vicente, 1999). A continuación, se desarrollaron un grupo de trabajos que inciden principalmente en describir herramientas y modelos para su aplicación (Escorsa \& Maspons, 2001; Miller, 2001; Morcillo, 2003). Más cercanos en el tiempo 
y en España, a partir de la publicación de la UNE 166006EX:2006, se desarrollan una serie de trabajos que se centran en explicar la citada norma y aportar guías y modelos de aplicación compatibles con el estándar (Benavides \& Quintana, 2006; Vergara, 2006). En esta línea, los trabajos más recientes se caracterizan por realizar casos de estudio sobre la aplicación de IC en grandes empresas, sectores y en el caso español también en centros de investigación (Rey Vázquez, 2006; Montes \& Lloveras, 2009; Aspinall, 2011; Lan, 2012; Tabor, 2012; Donohue \& Murphy, 2016).

Por lo tanto, el interés por la IC ha evolucionado de las etapas de definición propias de los primeros periodos de estudio, a las de descripción de modelos de aplicación, para centrarse de forma más reciente en los casos de aplicación y análisis en grandes empresas y centros públicos y/o de investigación, primando cada vez un enfoque más práctico.

En este sentido, el análisis de la literatura pone de manifiesto que es necesario avanzar en el estudio de la IC y que el siguiente paso en el desarrollo de la IC dentro del ámbito académico y profesional debería ser el análisis de su aplicación generalizada a todo tipo de organizaciones, en especial pymes donde hay una mayor carencia de trabajos, y con especial atención a evaluar los resultados derivados de aplicar IC (Antia \& Hesford, 2007; Brody, 2008; Herring \& Leavitt, 2011; Bulger, 2013; Sewdass \& Du Toit, 2014; Köseoglu, Ross \& Okumus, 2015). Cuestiones que precisamente trata de atender este trabajo en los siguientes apartados.

\section{Aproximación a los efectos de la IC en las organizaciones e hipótesis de investigación}

Como se ha comentado en la revisión de la literatura, el análisis de la literatura relativa a la medición de los efectos que la IC genera a nivel de empresa se centra en estudiar y describir casos individuales de grandes empresas. Sin embargo, en el caso de este trabajo el enfoque que se sigue difiere de lo anterior y busca analizar los efectos que la IC genera en una muestra de pymes. Para ello, en primer lugar se deben identificar los efectos que la literatura atribuye a la utilización de la IC en las empresas. En este sentido de la revisión de la literatura se identifican que los efectos más repetidos atribuibles a la IC son la capacidad de adaptación al entorno y la capacidad de innovar (Tena \& Comai, 2004; Rey Vázquez, 2006; Aspinall, 2011; AENOR, 2011; Köseoglu et al., 2015; Donohue \& Murphy, 2016). Además, consideramos que si la IC afecta a la capacidad de adaptación al entorno de una empresa y a su capacidad de innovación también cabría esperar un efecto positivo sobre sus resultados económicos (Yin, 2015; Soto-Acosta, Placer-Maruri \& Pérez-González, 2016). 
Por lo tanto, en coherencia con los puntos anteriores las hipótesis que se plantean en este trabajo son las siguientes:

H1. La utilización de IC por parte de las pymes ejerce un efecto positivo sobre su capacidad de adaptación al entorno.

H2. La utilización de IC porparte de las pymes ejerce un efecto positivo sobre su capacidad de innovar.

H3. La utilización de IC porparte de las pymes ejerce un efecto positivo sobre su rentabilidad económica.

\section{Metodología de la investigación}

Analizado el marco teórico y especificadas las hipótesis de la investigación, a continuación se definen las cuestiones metodológicas claves para el desarrollo del trabajo como la muestra objeto de estudio, variables y técnicas de análisis.

En relación a la muestra, se centra la atención en analizar los efectos generados por la utilización de IC en las pymes industriales de Cantabria. La elección de las pymes como objeto de investigación se debe a que pese a la importancia fundamental que tienen en nuestras economías, constituyendo el 99.9\% del entramado empresarial español y el 99.8 \% en la Unión Europea (Dirección General de Industria y de la PYME, 2015; European Comission, 2015), existen escasas aportaciones conceptuales y empíricas en la literatura que aborden, en concreto, los efectos que la utilización de IC genera en las pymes (Blenkhorn \& Fleisher, 2007; Gaidelys \& Meidute, 2012; Lan, 2012; Donohue \& Murphy, 2016).

La elección del sector industrial viene motivada porque al igual que en otras regiones de España y de Europa, en Cantabria ha sido un sector que ha ido perdiendo peso en la economía, especialmente durante la actual crisis, convirtiéndose las pymes industriales en una tipología de empresas que necesita políticas de apoyo y medidas que ayuden a mejorar su competitividad (OECD, 2011; Cámara de Comercio de Cantabria, 2014; European Comission, 2015).

Como se muestra en la Tabla 2 que recoge la ficha técnica de la investigación, el trabajo de campo de la investigación se desarrolló entre los meses de enero y septiembre del 2015 y el ámbito geográfico en el que se desarrolló fue la región de Cantabria, por ser el ámbito geográfico de trabajo más conocido por los autores, lo que facilita poder entrar en contacto con las empresas y, por tanto, aumentar las posibilidades de obtener mayor número de respuestas. La recogida y obtención de información se 
realizó mediante un primer contacto por correo electrónico explicando el objetivo del trabajo y un segundo correo electrónico que incluía un cuestionario dirigido al gerente o director general de la empresa. El listado de empresas al que contactar se obtuvo formando una base de datos que integrara los datos de varios directorios no homogéneos como los listados de la Cámara de Comercio (2014) y el Instituto Cántabro de Estadística (ICANE, 2014).

\begin{tabular}{|l|l|}
\hline Universo & $\begin{array}{l}437 \text { Pymes del Sector industrial con más de un empleado, forma societaria y } \\
\text { actividad ininterrumpida entre los años } 2009 \text { y 2014, ambos incluídos. }\end{array}$ \\
\hline Ámbito geográfico & Comunidad Autónoma de Cantabria \\
\hline Procedimiento de muestreo & Alcance a todo la población (enviados 437 cuestionarios) \\
\hline Recogida de Información & Encuesta enviada al gerente por correo electrónico \\
\hline Tamaño de la muestra & 79 Encuestas válidas \\
\hline Error muestral & $9.9 \%$ \\
\hline Nivel de confianza & $95.5 \%(\mathrm{~K}=1,96)$ para el caso más desfavorable $\mathrm{p}=\mathrm{q}=0.5$ \\
\hline Trabajo de campo & Enero-septiembre de 2015 \\
\hline
\end{tabular}

Tabla 2. Ficha técnica de la investigación

En cuanto al periodo temporal de análisis, indicar que al tratarse la IC de un proceso de gestión de información que afecta a decisiones estratégicas se debe considerar que no tiene por qué producir efectos inmediatos, considerándose necesario analizar periodos temporales de entre tres y cinco años (van Ark, Gupta, \& Erumban, 2011; Trigueros-Preciado, Pérez-Gonzalez, Solana-González, 2013; Pérez-Gonzalez \& Díaz-Díaz, 2015). Además y en relación a lo anterior, parecen adecuados estudios longitudinales, puesto que las variables explicadas medidas de forma transversal se pueden ver influidas por múltiples factores coyunturales. En este sentido, en nuestro trabajo, las variables dependientes son medidas como evolución de lo acontecido en los últimos cinco años.

Respecto a la definición de las variables utilizadas en la investigación y comenzando por la variable independiente, indicar que las investigaciones precedentes que analizan los efectos de las IC en las empresas son casos de estudio y lo hacen considerando como variable explicativa la utilización de IC. En nuestro caso dado que no estamos en un caso de estudio y que en la muestra puede haber empresas que si apliquen IC y otras que no, se considera dicha variable pero adaptándola y convirtiéndola en variable dicotómica que puede tomar dos valores, 0 si las empresas contestan que no aplican IC y 1 si contestan que si utilizan la IC. Se debe indicar que antes de que las empresas contestaran a esta pregunta, en el cuestionario se explica detalladamente a las empresas en que consiste la IC y cuando se puede considerar que una empresa aplica IC. En dicha explicación, en coherencia con da definición dada en la revisión de la literatura, se destaca que para considerar que una empresa aplica IC esta tiene 
que desarrollar de forma sistemática y continúa en el tiempo un proceso que conlleva todas y cada una de las siguientes fases: búsqueda activa de información en el exterior de la empresa y su filtrado y análisis para ponerla a disposición de los directivos y mejorar su toma de decisiones. Además, se contactó personalmente por vía telefónica con las empresas que manifestaron su deseo de participar en la investigación, para verificar si se habían entendido correctamente las cuestiones planteadas en el cuestionario y dar las explicaciones correspondientes en caso de que fuera necesario. En la Tabla 3 se puede ver las empresas agrupadas según su utilización de la IC.

\begin{tabular}{|c|r|r|r|}
\hline Utilización de la IC & \multicolumn{1}{|c|}{ N } & \multicolumn{1}{|c|}{$\%$} & \% acumulado \\
\hline No utilizan IC & 56 & $71 \%$ & 51 \\
\hline Si utilizan IC & 23 & $29 \%$ & 100 \\
\hline Total & 79 & 100 & 100 \\
\hline
\end{tabular}

Tabla 3. Distribución de frecuencias de la variable utilización de las TI

En cuanto a las variables dependientes, como ya se ha indicado serán la capacidad de adaptación (Tabla 4), que se refiere a la capacidad de anticipación y adaptación que la empresa tiene al entorno, la capacidad de innovar (Tabla 5) y los resultados económicos. Para la capacidad de anticipación al entorno se ha creado una escala de medida basada en los trabajos de Aspinall (2011), Sewdass y Du Toit (2014) y Köseoglu et al. (2015).

\begin{tabular}{|l|l|}
\hline Nombre abreviado & \multicolumn{1}{c|}{ Atributos } \\
\hline CAD 1 & El grado de satisfacción con la información que tiene del entorno es buena \\
\hline CAD 2 & Con la información que tiene puede anticiparse a las contingencias antes de que ocurran \\
\hline CAD 3 & Su capacidad de anticipación y reacción a los cambios del entorno es buena \\
\hline CAD 4 & Considera que se ha adaptado con rapidez a los cambios \\
\hline
\end{tabular}

Tabla 4. Escala formada como indicador de la capacidad de adaptación

Respecto a la capacidad de innovación se ha seguido la escala utilizada en la encuesta de innovación del INE (2014), por ser la más conocida para las pymes españolas al estar obligadas a su cumplimentación anual. 


\begin{tabular}{|l|l|}
\hline Nombre abreviado & \multicolumn{1}{c|}{ Atributos } \\
\hline CINNO 1 & Ha desarrollado mejoras en sus productos/servicios \\
\hline CINNO 2 & Ha desarrollado productos nuevos/servicios \\
\hline CINNO 3 & Ha desarrollado mejoras en sus procesos en el último año \\
\hline CINNO 4 & Ha desarrollado nuevos procesos \\
\hline
\end{tabular}

Tabla 5. Escala formada como indicador de los procesos internos

Por último, siguiendo trabajos previos la variable más utilizada para la medición de los resultados económicos es la rentabilidad económica (Powelt \& Dent-Micallef, 1997; Dedrick et al., 2003; Kohli \& Grover, 2008), en nuestro trabajo se planteó como la valoración que los directivos tenían sobre la evolución de la rentabilidad económica (ROI) de la empresa en los últimos cinco años. Además, si bien las dos primeras escalas están basadas en trabajos previos recogidos por la literatura, a efectos de garantizar su fiabilidad se calculó su alpha de Crombach obteniéndose valores superiores a 0.7 y por tanto satisfactorios (Hair, Black, Babin, Anderson \& Tatham, 2006).

Una vez obtenidos los indicadores definitivos por área de creación de valor, para cada una de las variables se generan modelos ANOVA, a partir de la división de las empresas de la muestra en dos grupos uno con las empresas que no utilización la IC, y otro formado por las empresas que si utilizan y tienen integrado como habitual la IC que lo que permitirá comprobar la existencia de efectos estadísticamente significativos entre la utilización de IC realizada por las organizaciones -variables independientes de los contrastes- y las escalas de las variables mencionadas que actúan como variables dependientes de los contrastes-.

\section{Resultados}

A continuación se recogen los resultados obtenidos en cada una de las variables mediante la presentación de las escalas utilizadas y una tabla que recoge, para cada grupo de empresas según su utilización de la IC, los valores medios, el valor del estadístico F y la significatividad del contraste, tanto a nivel global de la variable, promedio de los ítems que la integran, como individual, mediante distintos modelos ANOVA, uno para cada uno de los atributos que componen la variable. 


\subsection{Efecto de la utilización de la IC sobre la capacidad de adaptación al entorno}

En primer lugar, se presentan las tablas con los contrastes relativos a la escala capacidad de adaptación que se mostró en el apartado anterior (Tabla 4). En este sentido, se observa en las Tablas 6 y 7 que los resultados muestran la obtención de un efecto positivo y estadísticamente significativo tanto a nivel global de la variable, como para los 4 atributos que la componen. Esto, junto con la dirección positiva indicada por las medias, tanto para la variable global como para todos los atributos que la integran, permite confirmar la existencia de una relación positiva entre la utilización de la IC y la capacidad de adaptación al entorno de las pymes, de manera que las pymes que utilizan IC tiene mayor capacidad de adaptación al entorno.

\begin{tabular}{|c|c|c|}
\hline Utilización de la IC & \multicolumn{2}{|c|}{ Media "Capacidad de adaptación al entorno" } \\
\hline Sí Utilizan IC & 6.100 & \\
\hline No Utilizan IC & 3.350 & \\
\hline & Valor de la F & 6.073 \\
\hline & Significatividad & $0.009 * *$ \\
\hline
\end{tabular}

Tabla 6. Resultados ANOVA utilización de las TI y perspectiva global de clientes

\begin{tabular}{|c|c|c|c|c|c|}
\hline Utilización de la IC & \multicolumn{2}{|c|}{ (Medias) CAD 1} & CAD 2 & CAD 3 & CAD 4 \\
\hline Sí Utilizan IC & & 5.963 & 5.815 & 6.209 & 5.981 \\
\hline \multirow{3}{*}{ No Utilizan IC } & & 4.400 & 3.265 & 4.350 & 4.665 \\
\hline & Valor de la F & 5.776 & 9.830 & 6.129 & 4.502 \\
\hline & Significatividad & $0.007 * * *$ & $0.005^{* * *}$ & $0.009 * * *$ & $0.015^{* *}$ \\
\hline
\end{tabular}

Tabla 7. Resultados ANOVA utilización de la IC y atributos de la capacidad de adaptación al entorno

\subsection{Efecto de la utilización de la IC sobre la capacidad de innovación}

La existencia de relación entre la utilización de la IC y la variable dependiente capacidad de innovar representada con la escala mostrada en el apartado de metodología (Tabla 5), se mide a nivel global e individual. En este sentido a nivel global en la Tabla 8 se recoge los resultados, donde se puede observar que hay una relación estadísticamente significativa entre la utilización de la IC y la variable global capacidad de innovación. 


\begin{tabular}{|c|c|c|}
\hline Utilización de la IC & \multicolumn{2}{|c|}{ Media "capacidad de innovación" } \\
\hline Sí Utilizan IC & 5.715 & \\
\hline No Utilizan IC & 4.112 & \\
\hline & Valor de la F & 5.660 \\
\hline & Significatividad & $0.012^{* *}$ \\
\hline
\end{tabular}

Tabla 8. Resultados del ANOVA utilización de la IC a nivel global y capacidad de innovar

En lo que se refiere al análisis individual, en la Tabla 9 se observa que existe relación estadísticamente significativa para los 4 atributos. De forma que el grupo formado por las empresas que utilizan IC presenta una mayor capacidad de innovación que las que no utilizan IC.

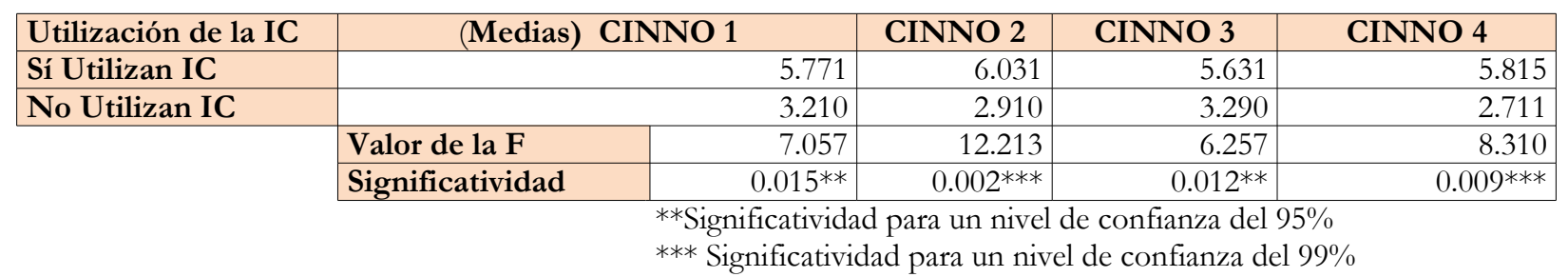

Tabla 9. Resultados ANOVA utilización IC y atributos de la capacidad de innovar

\subsection{Efecto de la utilización de las TI sobre los resultados económicos}

Por último, analizados los efectos de utilizar IC sobre la capacidad de adaptarse al entorno y la capacidad de innovar se procede a estudiar la existencia de relación entre la utilización de la IC y la evolución en el tiempo de la rentabilidad económica del negocio (ROI). La Tabla 10 recoge, como en los casos anteriores, los resultados del modelo ANOVA, indicando para cada grupo según utilización de la IC los valores medios, el valor de la F y la significatividad.

\begin{tabular}{|l|l|r|}
\hline Utilización de la IC & \multicolumn{2}{|c|}{ (Medias) ROI } \\
\hline Sí Utilizan IC & \multicolumn{2}{|c|}{1.580} \\
\hline No Utilizan IC & \multicolumn{2}{|c|}{0.735} \\
\hline \multirow{2}{*}{} & Valor de la F & 5.702 \\
\cline { 2 - 3 } & Significatividad & $0.012^{* *}$ \\
\cline { 2 - 3 } \\
\cline { 2 - 3 }
\end{tabular}

Tabla 10. Resultados del ANOVA utilización de la IC y resultados económicos 
Como se observa en la tabla anterior, la utilización de la IC tiene una relación directa, positiva y estadísticamente significativa con el ROI. De forma que las empresas que utilizan IC poseen a su vez una mejor evolución del ROI, contrastándose que existe un efecto positivo de la utilización de la IC sobre la rentabilidad económica de las pymes.

\section{Conclusiones}

Desde hace tiempo estamos inmersos en una tendencia de creciente complejidad de los contextos económicos y tecnológicos en los que nuestras empresas desarrollan su actividad, donde la capacidad de adaptación al entorno adquiere una importancia crucial y la innovación es reconocida como base sostenible de la competitividad de las organizaciones. Es en estos contextos donde la IC tiene un papel fundamental como proceso sistemático que detecta y analiza todas las informaciones del entorno susceptibles de afectar a la empresa y las pone a disposición de los directivos para apoyar su toma de decisiones.

El análisis de la literatura académica y profesional en relación a la IC, nos muestra por un lado que su estudio ha evolucionado de las etapas de definición propias de los primeros periodos de análisis, a las de descripción de sus posibles modelos de aplicación y certificación, para llegar a los trabajos más recientes centrados en casos de estudio y aplicación en grandes empresas y centros públicos o de investigación, primando cada vez un enfoque más práctico. En este sentido, la literatura reconoce la necesidad de avanzar en el estudio de la IC señalando como temas prioritarios extender su análisis y utilización a todo tipo de organizaciones y la obtención de métricas que permitan evaluar los resultados derivados de su aplicación.

Ante la situación descrita, este trabajo se centra en el estudio de los efectos de la IC en 79 pymes industriales con el objetivo de analizar los resultados que se derivan de la aplicación de la IC en estas organizaciones.

En este sentido podemos concluir que la IC como proceso de información que detecta, analiza y pone en conocimiento de la dirección todas aquellas cuestiones del entorno que pueden afectar a la empresa, favorece claramente el logro de una mayor capacidad de adaptación al entorno. Además, la IC en las pymes, al igual que ya había sido recogido en estudios de casos para grandes empresas, apoya la capacidad de innovación de las empresas, al aportar información sobre lo que hacen los competidores, lo que demandan los clientes, el estado de la técnica, lo que facilita el desarrollar nuevos o mejorados 
productos y servicios y nuevos y mejorados procesos. Algo que en el entorno industrial tiene una transcendencia fundamental.

Junto a lo anterior, el trabajo nos permite concluir que la IC además de afectar positivamente a la capacidad de adaptarse al entorno y a la capacidad de innovación, tiene efectos positivos sobre variables de tipo económico como es la evolución en el tiempo del ROI generado por las pymes.

Para finalizar, no se debe olvidar que este trabajo se ha centrado en 79 pymes del sector industrial y si bien los resultados de la investigación aquí presentados son significativos para las empresas de la muestra, estos se deben considerar con cautela en cuanto a su generalización a la totalidad de empresas industriales. En este sentido, aunque este trabajo es pionero en la medición de los resultados de la IC en una muestra de pymes, será necesario como futura línea de trabajo ampliar el número de empresas de la muestra, además de replicar este trabajo en otros sectores de actividad y analizar la inclusión de nuevas variables de estudio.

\section{Referencias}

AENOR (2011). Norma UNE 166006:2011 Gestión de la I+D+i: Sistema de vigilancia tecnológica e inteligencia competitiva.

Antia, K.D., \& Hesford, J.W. (2007). a Process-Oriented View of Competitive Intelligence and its Impact on Organizational Performance. Journal of Competitive Intelligence and Management, 4(1), 3-31.

Aspinall, Y. (2011). Competitive intelligence in the biopharmaceutical industry: The Key elements. Business Information Review, 28(2), 101-104. http://dx.doi.org/10.1177/0266382111411070

Attaway, M.C. (1998). A review of issues related to gathering and assessing competitive intelligence. American Business Review, 16(1), 25-35.

Benavides, C, \& Quintana, C. (2006). Inteligencia Competitiva, prospectiva e innovación. La norma UNE 166006 EX sobre el sistema de vigilancia tecnológica. Boletin Economico de ICE, 2896, 47-61.

Blenkhorn, D.L., \& Fleisher C.S. (2007). Performance Assessment in Competitive Intelligence: An Exploration, Synthesis, and Research Agenda. Journal of Competitive Intelligence and Management, 4(2), 4-22.

Brody, R. (2008). Issues in Defining Competitive Intelligence: An Exploration. Journal of Competitive Intelligence and Management, 4(3), 3-16. http://dx.doi.org/10.1109/emr.2008.4648884 
Bulger, N. (2013). Competitive Intelligence Professional Growth. Strategic and Competitive Intelligence Professionals, 5(4), 57-68.

Calof, J.L., \& Wright, S. (2008). Competitive intelligence: A practitioner, academic and inter-disciplinary perspective. European Journal of Marketing, 42(7/8), 717-730. http://dx.doi.org/10.1108/03090560810877114

Cámara de Comercio de Cantabria (2014). Informes de Coyuntura Industrial de Cantabria, IV Trimestre 2014. Disponible online en: http://www.camaracantabria.com/. (Fecha de último acceso: enero, 2015).

Cleland, D.I., \& King, W.R. (1975). Competitive business intelligence systems. Business Horizons, 18(6), 19-28. http://dx.doi.org/10.1016/0007-6813(75)90036-1

Chen, H., Chan, M., \& Zeng, D. (2002). CI Spider: A tool for competitive intelligence on the web. Decision Support Systems, 34, 1-17. http://dx.doi.org/10.1016/S0167-9236(02)00002-7

Dedrick, J., Gurbaxani, V., \& Kraemer, K.L. (2003). Information technology and economic performance: A critical review of the empirical evidence. ACM Computing surveys, 35(1), 1-28. http://dx.doi.org/10.1145/641865.641866

Dirección General de Industria y de la Pyme (2015). Retrato de las Pyme 2015. Disponible online en: http://www.ipyme.org/Publicaciones/Retrato PYME 2015.pdf. (Fecha de último acceso: enero, 2015).

Donohue, D.P., \& Murphy, P.M. (2016). Supporting Competitive Intelligence at DuPont by Controlling Information Overload and Cutting Through the Noise. Journal of Information \& Knowledge Management (online bajo pago). http://dx.doi.org/10.1142/S0219649216500040

Escorsa, P., \& Maspons, R. (2001). De la Vigilancia Tecnológica a la Inteligencia Competitiva. Madrid: Prentice Hall.

European Comission (2015) Innovation Union Scoreboard 2015. Disponible online en: http://ec.europa.eu/growth/industry/innovation/facts-figures/scoreboards/files/ius-2015 en.pdf

Eurofound, Agency of the European Union (2013). Report, Restructuring in SMEs in Europe. Disponible online en: www.eurofound.europa.eu/publications/htmlfiles/ef1247.htm

Gaidelys, V., \& Meidute, I. (2012). Instruments and methods of competitive intelligence. Economics and Management, 17(3), 971-977. http://dx.doi.org/10.5755/j01.em.17.3.2122

Gile, K., Kirby, J.P., Karel, R., Teubner, C., Driver, E., \& Murphy, B. (2006). Topic overview: Business intelligence. Retrieved from Forrester database.

Ghoshal, S., \& Westney, D.E. (1991). Organizing competitor analysis systems. Strategic Management Journal, 12(1), 17-31. http://dx.doi.org/10.1002/smj.4250120103 
Hair, J.F., Black, W.C., Babin, B.J., Anderson, R.E., \& Tatham, R.L. (2006). Multivariate data analysis. Upper Saddle River, NJ: Pearson Prentice Hall.

Herring, J.P., \& Leavitt, J. (2011). The Roadmap to a World-Class Competitive Intelligence Program. Competitive Intelligence, 14(1), 9-28.

ICANE, Instituto Cántabro de Estadística (2014). Encuesta industrial de empresas de Cantabria 2014. Disponible online en: http://www.icane.es/. (Fecha de último acceso: enero, 2015).

INE, Instituto Nacional de Estadística (2014). Disponible online en: http://www.ine.es/dynt3/metadatos/es/RespuestaDatos.htm?oe $=30061$

Kohli, R., \& Grover, V. (2008). Business Value of IT: An Essay on Expanding Research Directions to Keep up with the Times. Journal of the association for information systems, 9(1), 15-42.

Köseoglu, M.A., Ross, G., \& Okumus, F. (2015). Competitive intelligence practices in hotels. International Journal of Hospitality Management, 53, 161-172. http://dx.doi.org/10.1016/j.ijhm.2015.11.002

Lan, M.A. (2012). Research on the Utilization of Competitive Intelligence in Science and Technology Periodical Publication. Information Science, 1, 5-17.

Luu, T.T. (2013). Leading to learning and competitive intelligence. The Learning Organization, 20(3), 216-239. http://dx.doi.org/10.1108/09696471311328460

Maltz, E., \& Kohli, A.K. (1996). Market Intelligence dissemination across funtional boundaries. Journal of Marketiong Research, 35(1), 47-61. http://dx.doi.org/10.2307/3152012

Miller, S.H. (2001). Competitive Intelligence an overview. Competitive Intelligence Magazine, 1(11), 1-14.

Montes, J., \& Lloveras, J. (2009). La vigilancia tecnológica e inteligencia competitiva como proceso sistemático para la gestión de la información y la innovación en los centros de innovación y tecnología españoles. En: Semana Iberoamericana-Mediterranea del Desarrollo basado en el Conocimiento DBC Alicante, 1-20.

Morcillo, P. (2003). De la vigilancia e inteligencia competitiva a la creación de conocimientos. Madrid. Diponible online en: http://www.madrimasd.org/revista/revista17/tribuna/tribuna1.asp. (Fecha de último acceso: Febrero, 2015).

OCDE (2009). The impact of the global crisis on SME and entrepreneurship financing and policy responses. In Contribution to the OECD Strategic Response to the Financial and Economic Crisis, France 2009. 
OECD (2011). España Politicas para una Recuperación Sostenible. Disponible online en: http://www.oecd.org/spain/44660757.pdf

OECD (2015). Taxation of SMEs in OECD and G20 Countries 2015. DOI : 10.1787/9789264243507-en

Palop, F., \& Vicente, J.M. (1999). Vigilancia Tecnológica. Valencia: Fundación COTEC, Estudio nº 14.

Pearce, F.T. (1976). Business Intelligence Systems: The need, development, and integration. Industrial Marketing Management, 5(2/3), 115-138. http://dx.doi.org/10.1016/0019-8501(76)90035-3

Pérez-González, D., \& Díaz-Díaz, R. (2015). Public Services Provided with ICT in the Smart City Environment: The Case of Spanish Cities. Journal of Universal Computer Science, 21(2), 248-267.

Prescott, J.E. (1989). Competitive Intelligence: Its Role and Function in Organizations. Advances in Competitive Intelligence. Vienna: J.E. Prescott (Ed.).

Powelt, T., \& Dent-Micallef, A. (1997). Information Technology as Competitive Advantage: The Role of Human, Business and Technology Resources. Strategic Management Journal, 18(5), 375-405. http://dx.doi.org/10.1002/(SICI)1097-0266(199705)18:5<375::AID-SMJ876>3.3.CO;2-Z

Rey Vázquez, L. (2006). Ferroatlántica I+D y la vigilancia tecnológica. El profesional de la Información, 15(6), 420-425. http://dx.doi.org/10.3145/epi.2006.nov.03

Sewdass, N., \& Du Toit, A. (2014). Current state of competitive intelligence in South Africa. International Journal of Information Management, 34(2), 185-190.

http://dx.doi.org/10.1016/j.ijinfomgt.2013.10.006

Soto-Acosta, P., Placer-Maruri, E., \& Pérez-González, D. (2016). A case analysis of a product lifecycle information management framework for SMEs. International Journal of Information Management, 36(2), 240-244. http://dx.doi.org/10.1016/j.ijinfomgt.2015.12.001

Tabor, E. (2012). Review of Pharmaceutical Competitive Intelligence for the Regulatory Affairs Professional. Drug Information Journal, 46(6), 745-746. http://dx.doi.org/10.1177/0092861512460760

Tena, J., \& Comai, A. (2004). La Inteligencia Competitiva en las Multinacionales Catalanas. Barcelona, EMECOM.

Trigueros-Preciado, S., Pérez-González, D., \& Solana-González, P. (2013). Cloud computing in industrial SMEs: Identification of the barriers to its adoption and effects of its application. Electronic Markets, 23(2), 105-114. http://dx.doi.org/10.1007/s12525-012-0120-4

Vergara, J. (2006). La Vigilancia Tecnológica antes y después de la UNE 166006:2006 EX. Puzzle: Revista de Inteligencia Competitiva, 5(22), 37-41. 
Yin, C.Y. (2015). Measuring organizational impacts by integrating competitive intelligence into executive information system. Journal of Intelligent Manufacturing,1-15 (online).

http://dx.doi.org/10.1007/s10845-015-1135-4

Intangible Capital, 2016 (www.intangiblecapital.org)

Article's contents are provided on an Attribution-Non Commercial 3.0 Creative commons license. Readers are allowed to copy, distribute and communicate article's contents, provided the author's and Intangible Capital's names are included. It must not be used for commercial purposes. To see the complete license contents, please visit http://creativecommons.org/licenses/by-nc/3.0/. 